\title{
Symmetry Protection of Photonic Entanglement in the Interaction with a Single Nanoaperture
}

\author{
Alexander Büse, ${ }^{1, *}$ Mathieu L. Juan, ${ }^{2,3}$ Nora Tischler, ${ }^{4}$ Vincenzo D’Ambrosio, ${ }^{5,6}$ \\ Fabio Sciarrino, ${ }^{7}$ Lorenzo Marrucci, ${ }^{6}$ and Gabriel Molina-Terriza ${ }^{8,9, \dagger}$ \\ ${ }^{1}$ Department of Physics \& Astronomy, Macquarie University, NSW 2109 Sydney, Australia \\ ${ }^{2}$ Institute for Quantum Optics and Quantum Information of the Austrian Academy of Sciences, A-6020 Innsbruck, Austria \\ ${ }^{3}$ Institute for Experimental Physics, University of Innsbruck, A-6020 Innsbruck, Austria \\ ${ }^{4}$ Centre for Quantum Dynamics, Griffith University, Brisbane 4111, Australia \\ ${ }^{5}$ ICFO-Institut de Ciencies Fotoniques, The Barcelona Institute of Science and Technology, \\ E-08860 Castelldefels, Barcelona, Spain \\ ${ }^{6}$ Dipartimento di Fisica, Università di Napoli Federico II, 80126 Napoli, Italy \\ ${ }^{7}$ Dipartimento di Fisica, Sapienza Università di Roma, I-00185 Roma, Italy \\ ${ }^{8}$ Centro de Física de Materiales (MPC) and Donostia International Physics Center (DIPC), \\ 20018 Donostia-San Sebastin, Spain \\ ${ }^{9}$ IKERBASQUE, Basque Foundation for Science, 48013 Bilbao, Spain
}

(Received 3 April 2018; published 26 October 2018)

\begin{abstract}
In this work, we experimentally show that quantum entanglement can be symmetry protected in the interaction with a single subwavelength plasmonic nanoaperture, with a total volume of $V \sim 0.2 \lambda^{3}$. In particular, we experimentally demonstrate that two-photon entanglement can be either completely preserved or completely lost after the interaction with the nanoaperture, solely depending on the relative phase between the quantum states. We achieve this effect by using specially engineered two-photon states to match the properties of the nanoaperture. In this way we can access a symmetry protected state, i.e., a state constrained by the geometry of the interaction to retain its entanglement. In spite of the small volume of interaction, we show that the symmetry protected entangled state retains its main properties. This connection between nanophotonics and quantum optics probes the fundamental limits of the phenomenon of quantum interference.
\end{abstract}

DOI: $10.1103 /$ PhysRevLett.121.173901

The quantum properties of light can be used as a resource in applications such as communication, computation, and sensing. Quantum states of light have been harnessed to demonstrate most of the building blocks needed for quantum information processing [1] and more recently have been used in compact integrated waveguide arrays to perform simple quantum simulation tasks [2-4]. The maturity of quantum sources of light allows for a broad range of possibilities to control the features of light fields such as polarization, pulse duration, spatial modes, etc. However, most of the achievements in quantum photonics are hindered by the same limitations as the classical processing of light: weak interactions with matter, which impede efficient nonlinear processes, and large devices with dimensions many times the wavelength of light $(\lambda)$. Plasmonic devices may hold the key to overcome these hurdles due to strong interaction with light, small volumes of interaction, and the possibility to engineer and fabricate suitable nanostructures to address particular tasks. Classical control of the plasmonic modes of nanostructures has already been achieved by the use of modulation of ultrashort pulses [5], spatial control of the incident modes of light [6], and the use of different angular momentum modes
[7]. These achievements have allowed the processing of the classical properties of light at the nanoscale, the development of novel biosensors, and the enhancement of nonlinear processes for molecular characterization (see Ref. [8] for a review on the subject).

In the context of quantum optics, plasmonic waveguides have shown that they can interact strongly with singlephoton emitters and transport single photons [9]. Plasmonic Hong-Ou-Mandel (HOM) interferometers have been implemented by combining waveguides of several micrometers length to form beam splitters [10-13]. In this way it has been shown that quantum correlations of propagating photons can survive the interaction with plasmonic structures. A similar effect was observed in large arrays of nanoapertures [14] where the phenomenon of extraordinary optical transmission was exploited [15]. In all these works quantum light interacts with structures with a total size much larger than $\lambda$. In particular, in the seminal work of Ref. [14], it was shown that the entanglement of the photons did not survive when the photons were focused to one single nanoaperture. Thus, while quantum correlations and quantum entanglement have been observed to survive in structures with an overall size larger than $\lambda$, the 
fundamental question of whether photonic quantum entanglement can be processed or even survive the interaction in the subwavelength regime, remains open to the best of our knowledge.

There has also been a series of efforts to directly observe the quantum properties of the electronic coherent oscillations associated with plasmon resonances [16]. These observations open the way to quantum processing of the photonic information at the nanoscale, but they require understanding which quantum entangled states are most suitable for the interaction with isolated nanostructures.

Here we present experimental evidence that symmetry protected quantum entangled photonic states can interact with a single nanostructure with a total volume of $V \sim 0.2 \lambda^{3}$ without being affected. This approach is reminiscent of the recent efforts in quantum information aimed at utilizing decoherence-free subspaces (DFSs). Studied both theoretically [17] and experimentally $[18,19]$, DFSs usually make use of collective decoherence $[18,19]$ or the symmetry of the state [20]. Similarly to the photonic experiments on DFSs, in this work we focus on the postselected states after the interaction with the nanoparticle, where the two photons arrive at the detectors. In this way, we elude the detrimental effects of losses which cannot be avoided in plasmonic interactions.

We work with a simple nanostructure consisting of an isolated circular aperture. This kind of structure is versatile and has been used for nanotrapping experiments [21] and classical sensing of molecules [22], and is essential in near field optical microscopy experiments [23]. Our choice of the nanostructure was motivated by its high symmetry and the fact that it is well studied. Even though there is no analytical solution of the Maxwell equations for this structure, many interesting properties have been found both theoretically and experimentally. In particular, nanoapertures mix the polarization components of an incident classical field, an effect that can be described as spin-orbit coupling [24]. One way of explaining this phenomenon is in terms of symmetries: The coupling results from the mixing of the helicities of the field while total angular momentum is conserved. This helicity mixing naturally occurs in nondual structures, while the circular symmetry of the structure imposes the conservation of total angular momentum [25]. A light field of well-defined helicity can be decomposed into a series of plane waves all having the same circular polarization, and the helicity can then take on only two values [26]. Working with the total angular momentum and the helicity has the advantage that they remain valid in both paraxial and nonparaxial regimes allowing for an easy description of the experimental setup [24]. Interestingly, the helicity and angular momentum eigenvalues are conserved in the focusing with high numerical aperture microscope objectives [24,25,27], even if the electromagnetic fields are very different in the paraxial and focused regimes [7]. Therefore, the electromagnetic modes can be described with the same labels both in the paraxial and the focusing regimes.

As a consequence, a circular nanoaperture can simply be described as a (lossy) beam splitter where the modes are mixed in polarization - the two helicities - instead of being mixed into two different propagation directions. Any other structure with the same symmetries described here will essentially behave in the same way. Examples of this could be "bull's eye" corrugated apertures [28] or tapered nanofibers [29]. Then, for a single photon creation operator $\hat{a}_{m, \Lambda}^{\dagger}$ with total angular momentum $m$ and helicity $\Lambda$, the nanoaperture causes the following transformation $\hat{a}_{m, \Lambda}^{\dagger} \rightarrow$ $\alpha_{m, \Lambda} \hat{b}_{m, \Lambda}^{\dagger}+\beta_{m, \Lambda} \hat{b}_{m,-\Lambda}^{\dagger}+\sum_{i=\{1,2\}} \gamma_{m, \Lambda, i} \hat{c}_{m, i}^{\dagger}$. The probability amplitudes $\gamma_{m, \Lambda, i}$ summarize all the losses in the system (going to unwanted ancilla modes described by $\hat{c}_{m, \Lambda, i}^{\dagger}$ ) [30], while the modes $\hat{b}^{\dagger}$ represent the ones we can detect. The probability for the helicity flip depends on the relative strengths of $\alpha$ and $\beta$, when passing through the nanoaperture. Note that the total angular momentum is conserved, since the aperture is cylindrically symmetric. Postselecting the transmitted states, one can avoid the detrimental effect of losses due to light reflected from the metallic film, coupling to surface and localized plasmon modes and other losses in the metal. Therefore, in the following we will leave out the effect of the ancilla modes $(\hat{c})$. Then, even if the amplitudes $\alpha$ and $\beta$ can be normalized, the phases of the transmitted amplitudes are not locked as in the unitary beam splitter [31]. Nevertheless, the mirror symmetry of the structure imposes an important restriction on the amplitudes: $\alpha_{m, \Lambda}=\alpha_{-m,-\Lambda}$ and $\beta_{m, \Lambda}=\beta_{-m,-\Lambda}$. In particular, the subspace of modes with $m=0$ will transform onto itself:

$$
\hat{a}_{0,+}^{\dagger} \rightarrow \alpha \hat{b}_{0,+}^{\dagger}+\beta \hat{b}_{0,-}^{\dagger}, \quad \hat{a}_{0,-}^{\dagger} \rightarrow \alpha \hat{b}_{0,-}^{\dagger}+\beta \hat{b}_{0,+}^{\dagger},
$$

where the subindices in the amplitudes have been omitted for simplicity. These equations are similar to those of a twomode beam splitter and, consequently, we can exploit its properties to transform and process entangled photon states $[32,33]$. Thus, considering two photons, there are three linearly independent basis states possible within this subspace:

$$
\begin{aligned}
& \left|\Psi_{0}\right\rangle=\hat{a}_{0,+}^{\dagger} \hat{a}_{0,-}^{\dagger}|0\rangle \rightarrow \frac{1}{\sqrt{2}}\left(|R\rangle_{1}|L\rangle_{2}+|L\rangle_{1}|R\rangle_{2}\right), \\
& \left|\Psi_{+}\right\rangle=\frac{1}{2}\left(\hat{a}_{0,+}^{\dagger 2}+\hat{a}_{0,-}^{\dagger 2}\right)|0\rangle \rightarrow \frac{1}{\sqrt{2}}\left(|R\rangle_{1}|R\rangle_{2}+|L\rangle_{1}|L\rangle_{2}\right), \\
& \left|\Psi_{-}\right\rangle=\frac{1}{2}\left(\hat{a}_{0,+}^{\dagger 2}-\hat{a}_{0,-}^{\dagger 2}\right)|0\rangle \rightarrow \frac{1}{\sqrt{2}}\left(|R\rangle_{1}|R\rangle_{2}-|L\rangle_{1}|L\rangle_{2}\right) .
\end{aligned}
$$

After the arrow, we show the first quantization expression of the two-photon states, with $\hat{a}_{0,+/}^{\dagger}|0\rangle \rightarrow|R / L\rangle$ and the subindex $(1,2)$ labeling the photon. We use a detection 
method with a beam splitter, which directly measures the first quantized states [34]. When measuring the two-photon state in this way an extra state $(1 / \sqrt{2})\left(|R\rangle_{1}|L\rangle_{2}-|L\rangle_{1}|R\rangle_{2}\right)$ can appear. This state is not allowed by boson symmetrization, and it conveys information about the distinguishability of the photons. Thus, it is incoherent with the three other states [35].

The states $\left|\Psi_{+}\right\rangle$and $\left|\Psi_{-}\right\rangle$are two-photon entangled NOON states, which describe two-mode superpositions of $N$ photons, where all the photons are in one of the two modes. Polarization two-photon NOON states have already been used to beat the standard quantum limit of sensitivity in atomic spin species [36]. In this work, we show that by further using the symmetries of these states, one can control their interaction with nanostructures in highly focused systems.

The states $\left|\Psi_{0}\right\rangle$ and $\left|\Psi_{+}\right\rangle$are mirror symmetric relative to any mirror plane containing the beam axis, while $\left|\Psi_{-}\right\rangle$is the only mirror antisymmetric state within the whole subspace spanned by the three basis states. Owing to the symmetry of $\left|\Psi_{-}\right\rangle$, the state is protected in this system. This is a consequence of the fact that the interaction with the material system has to preserve the cylindrical symmetry of the photonic state and its mirror symmetry. Given that the aperture is cylindrically symmetric and consequently mirror symmetric, the Hamiltonian of interaction, complicated as it may be, must preserve these properties of the photonic states. As the $\left|\Psi_{-}\right\rangle$state is the only state of the set with a total angular momentum of zero and mirror antisymmetry, it cannot transform to any other state and it is protected against decoherence. So, even when both photons are transmitted through a circular aperture whose size is smaller than the wavelength, they should remain in this entangled state. This is clearly seen when applying the transformation (1) to the states $\left|\Psi_{-}\right\rangle$and $\left|\Psi_{+}\right\rangle$:

$$
\begin{aligned}
\left|\Psi_{-}\right\rangle \rightarrow & \frac{1}{2}\left(\alpha^{2}-\beta^{2}\right)\left(\hat{b}_{0,+}^{\dagger} \hat{b}_{0,+}^{\dagger}-\hat{b}_{0,-}^{\dagger} \hat{b}_{0,-}^{\dagger}\right)|0\rangle, \\
\left|\Psi_{+}\right\rangle \rightarrow & \frac{1}{2}\left(\alpha^{2}+\beta^{2}\right)\left(\hat{b}_{0,+}^{\dagger} \hat{b}_{0,+}^{\dagger}+\hat{b}_{0,-}^{\dagger} \hat{b}_{0,-}^{\dagger}\right)|0\rangle \\
& +2 \alpha \beta \hat{b}_{0,+}^{\dagger} \hat{b}_{0,-}^{\dagger}|0\rangle .
\end{aligned}
$$

Most notably, while the only difference between the two input states resides in the phase between the two quantum states contributing to the entanglement, the difference after the subwavelength aperture is dramatic: one state survives structurally unaffected, while $\left|\Psi_{+}\right\rangle$is mixed with $\left|\Psi_{0}\right\rangle$.

In order to study the two-photon state with and without the interaction with the nanoaperture, we design an experimental set-up as depicted in Fig. 1. The two-photon generation is performed through spontaneous parametric down conversion in a collinear configuration at a degenerate wavelength of $808.5 \mathrm{~nm}$ [34]. The maximum HOM interference visibility, attesting the two-photon indistinguishability, was found to be $\mathrm{V}=90 \%$ and it was limited by the frequency spectrum of the photons. The temporal overlap of the two generated photons was precisely

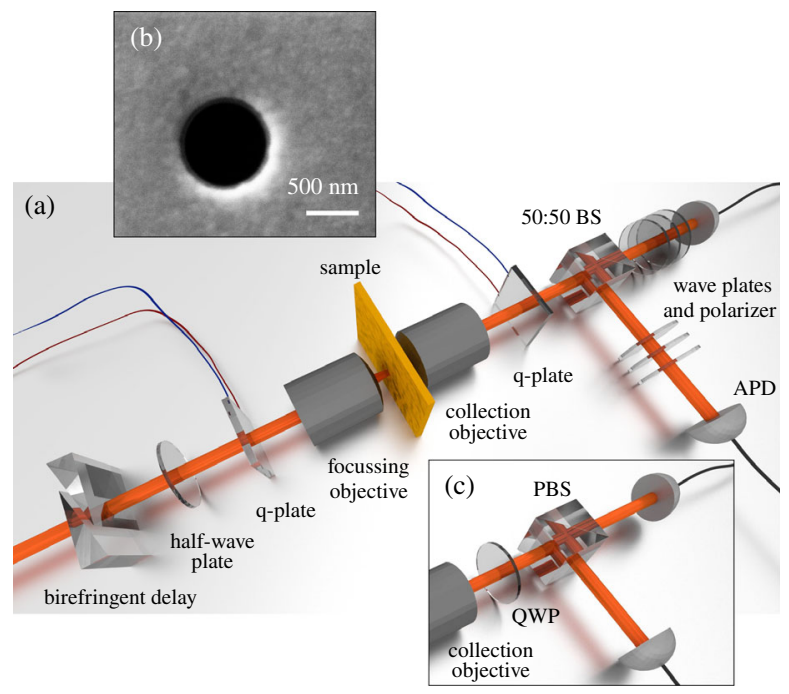

FIG. 1. (a) Schematic of the experimental set-up for transmission of protected quantum states through the nanoaperture. (b) Scanning electron microscope image of our target structure: a circular aperture of $750 \mathrm{~nm}$ diameter. Scale bar of length $500 \mathrm{~nm}$. (c) Variation of the set-up to directly measure the quantum interference signature. Details of the set-up can be found in the text.

controlled using a birefringent delay. A critical step in order to access the symmetry protected subspace for interaction with the nanoaperture is to transform the modes of the photons to those with total angular momentum zero. This step is achieved with a " $q$ plate" $[37,38]$ with $q=1 / 2$, which transforms our photons to the required modes. A half-wave plate placed before the $q$ plate allows us to select either the $\left|\Psi_{+}\right\rangle$or the $\left|\Psi_{-}\right\rangle$state [34]. The prepared twophoton state is then strongly focused with a microscope objective of numerical aperture $\mathrm{NA}=0.85$ and subsequently collected with a second microscope objective with $\mathrm{NA}=1.4$ to be finally analyzed.

In Figs. 2(a)-2(b) we present a tomographic reconstruction of the states in the absence of a nanoaperture in the system. As shown in Fig. 1, in order to tomographically analyze the photons we first transform them back to Gaussian modes with a $q$ plate reversing the effect of the initial one. The rest of the tomography is a simple polarization analysis as, ideally, the spatial modes of the photons are now identical. The two-photon state is postselected with a coincidence logic, after probabilistically splitting on a beam splitter. The reconstructed density matrices, $\rho$, show a fidelity with the $\left|\Psi_{+}\right\rangle$and the $\left|\Psi_{-}\right\rangle$, $F=\left\langle\Psi_{ \pm}|\rho| \Psi_{ \pm}\right\rangle$, of more than $60 \%$, limited by a weak incoherent contribution of the $\hat{a}_{+}^{\dagger} \hat{a}_{-}^{\dagger}|0\rangle$ modes. This loss of coherence is mainly due to the fact that we use bucket detectors to collect the photons, instead of projecting onto single mode fibers. Both $q$ plates provide the proper azimuthal transformation, but the second $q$ plate does not properly compensate for the radial profile of the photons. Also, slight misalignments and imperfections of 

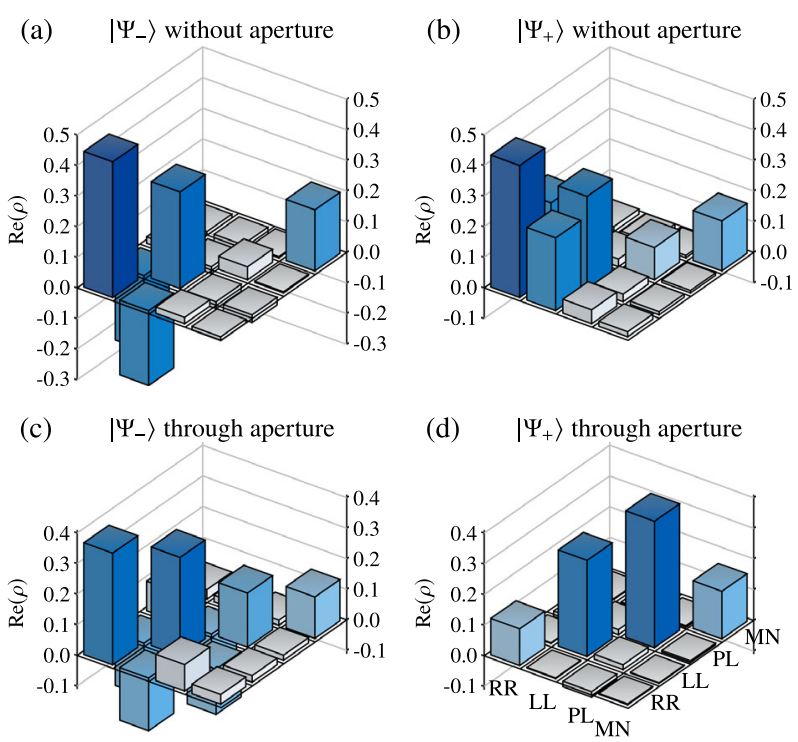

FIG. 2. Reconstructed real part of the post-selected density matrices. (a) and (c) $\left|\Psi_{-}\right\rangle$and (b) and (d) $\left|\Psi_{+}\right\rangle$incident states. (a)-(b) no nanoaperture present, (c)-(d) after interaction with the nanoaperture. The basis is given in terms of the modes arriving at the two detectors. Labels on the axis represent the following states: RR $|R R\rangle$, LL $|L L\rangle, \operatorname{PL}\left|\Psi_{0}\right\rangle$, MN $(1 / \sqrt{2})(|R L\rangle-|L R\rangle)$. The imaginary parts are consistent with being zero within experimental uncertainty.

the two $q$ plates result in the creation of higher-order transverse modes.

Once the two photons are allowed to interact with the nanoaperture, we perform another tomographic reconstruction of the states, as presented in Figs. 2(c)-2(d). Now, the difference between the $\left|\Psi_{-}\right\rangle$(minus) and $\left|\Psi_{+}\right\rangle$ (plus) states transformed through their interaction with the nanoaperture is remarkable. Where the minus state remains very similar to the state before the interaction [cf. Fig. 2(a)], with only slightly increased contributions from mixed polarization channels, the plus state [cf. Fig. 2(b)] has changed dramatically. The coherence between the $|L\rangle_{1}|L\rangle_{2}$ and $|R\rangle_{1}|R\rangle_{2}$ contributions has completely vanished, while mixed terms are as strong as the pure $|L\rangle_{1}|L\rangle_{2}$ and $|R\rangle_{1}|R\rangle_{2}$ contributions. Interestingly, some coherence between the $|L\rangle_{1}|R\rangle_{2}$ and $|R\rangle_{1}|L\rangle_{2}$ terms has emerged. This coherence is consistent with a mixing with the $\left|\Psi_{0}\right\rangle$ state, rather than misalignments, while the extra noise in all polarization channels is mainly due to the decreased signal to noise ratio after the interaction.

In Table I we quantify the effect on the entanglement of the interactions with the nanoaperture. We use two measures: the concurrence $[39,40]$ related to the entanglement of formation, and the negativity, which measures the entanglement that can be distilled [40]. In both cases, a value of $1(0)$ indicates a maximally entangled (separable) state. We find that both concurrence and negativity of the initial states indicate the presence of entanglement for both minus and plus states. However, although both states initially show the same degree of entanglement, after the interaction only the minus state maintains its degree of entanglement, whereas the plus state's entanglement has virtually vanished. The fidelity, comparing the overlap between the experimental and ideal states, shows a similar behavior: it is mostly preserved by the minus state through the interaction, whereas the fidelity of the plus state is greatly reduced, as is also obvious from directly comparing the density matrices in Figs. 2(b) and 2(d).

The signature of this dramatic difference between the transformation of the plus and minus states can be observed by replacing the tomographic reconstruction with a simple projection onto the circularly polarized states, followed by HOM type interference visibility measurements [see Fig. 1(c)]. A second $q$ plate is not needed, because projecting into the polarization states produces zero coincidences for the $\left|\Psi_{ \pm}\right\rangle$states [34]. In Fig. 3(a) the effect of the delay between the two photons on the preparation of the states can be observed.

Only when the two photons overlap in time, the operators in Eq. (3) are indistinguishable and produce the desired states. In this situation, the states $\left|\Psi_{ \pm}\right\rangle$attain a minimum in the HOM interference, as both go to the same port of the polarizing beam splitter. At nonzero delay, the photons are distinguishable and can randomly go to both ports of the polarizing beam splitter, raising the coincidence events [34]. On the other hand, the transformation to the state $\left|\Psi_{0}\right\rangle$ raises the coincidence count at zero delay. Therefore, when the photons do not interact with the nanoaperture, the states $\left|\Psi_{ \pm}\right\rangle$achieve the same visibility, while differences in the visibility may appear after the photons have interacted with the nanoaperture. In this latter situation, the transformation of the states can be distinguished and, while the $\left|\Psi_{-}\right\rangle$state retains a high visibility,

TABLE I. Comparison of entanglement related quantities between minus and plus states before and after the interaction with the nanoaperture.

\begin{tabular}{|c|c|c|c|c|}
\hline & \multicolumn{2}{|c|}{ No interaction } & \multicolumn{2}{|c|}{ Interaction with aperture } \\
\hline & $\left|\Psi_{-}\right\rangle$ & $\left|\Psi_{+}\right\rangle$ & $\left|\Psi_{-}\right\rangle$ & $\left|\Psi_{+}\right\rangle$ \\
\hline Concurrence & $0.253 \pm 0.009$ & $0.233 \pm 0.009$ & $0.220 \pm 0.048$ & $0.020 \pm 0.019$ \\
\hline Negativity & $0.253 \pm 0.009$ & $0.230 \pm 0.009$ & $0.201 \pm 0.044$ & $0.017 \pm 0.016$ \\
\hline Fidelity to $2^{-1 / 2}(|R R\rangle \pm|L L\rangle)$ & $0.624 \pm 0.004$ & $0.603 \pm 0.005$ & $0.515 \pm 0.024$ & $0.270 \pm 0.020$ \\
\hline
\end{tabular}




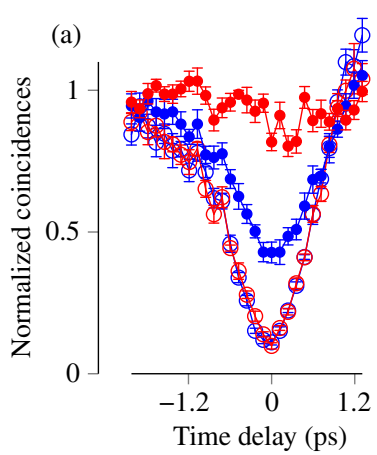

(b)

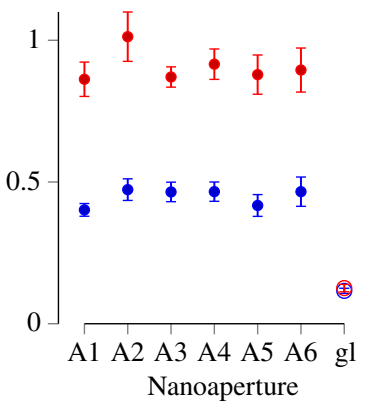

FIG. 3. (a) Normalized coincidence count rates for plus (red) and minus (blue) states as a function of the time delay between the two photons. Open markers represent the states going through the glass only, not interacting with the nanoaperture, while solid markers represent the effect after transmission through a particular nanoaperture. (b) Shows coincidences at zero delay for six different apertures (A1-A6) with nominally the same diameter and also when the states go through glass (gl). Each coincidence rate has been measured 10 times and the error bars span 1 standard deviation of the resulting distribution. The coincidences are normalized to the coincidences at very long delays (not shown in the figures).

the mirror symmetric state $\left(\left|\Psi_{+}\right\rangle\right)$completely loses its visibility. Then, the normalized coincidence rate at zero delay allows us to quickly and reliably analyze the interaction of the engineered states with a number of nanostructures on one sample. We compared several nanoapertures with identical nominal size, proving that the stark difference between the plus and minus states is robust to the variations in the fabrication process of the nanostructures, as shown in Fig. 3(b).

In this work we have demonstrated that despite the extreme changes the electromagnetic modes undergo through strong focusing and the interaction with the nanostructure, by exploiting the symmetries of the system it is possible to engineer entangled states that are protected against decoherence and unwanted transformations in the absence of losses. We have also demonstrated that this interaction strongly depends on the quantum phase between the entangled modes in such a way that a $\pi$ phase shift between the relative amplitudes of the states can be distinguished through the interaction with the nanostructures. From a fundamental point of view, this is a result of the important impact that this quantum phase has on the symmetry of the two-photon state. Such a difference survives in the subwavelength regime, opening a promising approach to study the intricate interplay between plasmonics and quantum optics. Indeed, the behavior of the $\left|\Psi_{+}\right\rangle$state and its deterioration through the interaction is rather puzzling and more complicated than what is distilled from Eq. (3). Looking at the density matrix of Fig. 2 (d), we observe the emergence of the state $\Psi_{0}$, which requires coherence between $|R\rangle_{1}|L\rangle_{2}$ and $|L\rangle_{1}|R\rangle_{2}$. However, there is no coherence with the initial state. One possible explanation of this phenomenon would be coupling to the localized or surface plasmon modes, which, due to the monogamy property of entanglement [41], would give rise to decoherence between the $\left|\Psi_{0}\right\rangle$ and $\left|\Psi_{+}\right\rangle$states. Nevertheless, the symmetry protected state $\left|\Psi_{-}\right\rangle$remains impervious to decoherence due to its unique geometrical properties.

L. M. and F. S. acknowledge financial support from the European Union Horizon 2020 program, within the European Research Council (ERC) Grant No. 694683, PHOSPhOR. G. M. T. acknowledges financial support from the Australian Research Council through the Future fellowship program FT110100924 and the Spanish Ministry of Science, Innovation and Universities (MICIN) Project No. FIS2017-87363-P.

*alexander.buese@mailbox.org 'gabriel.molina.terriza@gmail.com

[1] T. E. Northup and R. Blatt, Nat. Photonics 8, 356 (2014).

[2] J. O. Owens, M. A. Broome, D. N. Biggerstaff, M. E. Goggin, A. Fedrizzi, T. Linjordet, M. Ams, G. D. Marshall, J. Twamley, M. J. Withford, and A. G. White, New J. Phys. 13, 075003 (2011).

[3] J. Carolan, C. Harrold, C. Sparrow, E. Martín-López, N. J. Russell, J. W. Silverstone, P. J. Shadbolt, N. Matsuda, M. Oguma, M. Itoh, G. D. Marshall, M. G. Thompson, J. C. F. Matthews, T. Hashimoto, J. L. O'Brien, and A. Laing, Science 349, 711 (2015).

[4] M. Bentivegna, N. Spagnolo, C. Vitelli, F. Flamini, N. Viggianiello, L. Latmiral, P. Mataloni, D. J. Brod, E. F. Galvão, A. Crespi, R. Ramponi, R. Osellame, and F. Sciarrino, Sci. Adv. 1, e1400255 (2015).

[5] M. Aeschlimann, M. Bauer, D. Bayer, T. Brixner, F. J. Garcia de Abajo, W. Pfeiffer, M. Rohmer, C. Spindler, and F. Steeb, Nature (London) 446, 301 (2007).

[6] G. Volpe, G. Molina-Terriza, and R. Quidant, Phys. Rev. Lett. 105, 216802 (2010).

[7] X. Zambrana-Puyalto, X. Vidal, and G. Molina-Terriza, Nat. Commun. 5, 4922 (2014).

[8] J. N. Anker, W. P. Hall, O. Lyandres, N. C. Shah, J. Zhao, and R. P. Van Duyne, Nat. Mater. 7, 442 (2008).

[9] A. V. Akimov, A. Mukherjee, C. L. Yu, D. E. Chang, A. S. Zibrov, P. R. Hemmer, H. Park, and M. D. Lukin, Nature (London) 450, 402 (2007).

[10] R. W. Heeres, L. P. Kouwenhoven, and V. Zwiller, Nat. Nanotechnol. 8, 719 (2013).

[11] J. S. Fakonas, H. Lee, Y. A. Kelaita, and H. A. Atwater, Nat. Photonics 8, 317 (2014).

[12] G. Di Martino, Y. Sonnefraud, M. S. Tame, S. Kéna-Cohen, F. Dieleman, Ş. K. Özdemir, M. S. Kim, and S. A. Maier, Phys. Rev. Applied 1, 034004 (2014).

[13] Y.-J. Cai, M. Li, X.-F. Ren, C.-L. Zou, X. Xiong, H.-L. Lei, B.-H. Liu, G.-P. Guo, and G.-C. Guo, Phys. Rev. Applied 2, 014004 (2014).

[14] E. Altewischer, M. P. van Exter, and J. P. Woerdman, Nature (London) 418, 304 (2002).

[15] T. W. Ebbesen, H. J. Lezec, H. F. Ghaemi, T. Thio, and P. A. Wolff, Nature (London) 391, 667 (1998). 
[16] M. S. Tame, K. R. McEnery, Ş. K. Özdemir, J. Lee, S. A. Maier, and M. S. Kim, Nat. Phys. 9, 329 (2013).

[17] G. Massimo Palma, K.-A. Suominen, and A. K. Ekert, Proc. R. Soc. A 452, 567 (1996).

[18] P. G. Kwiat, A. J. Berglund, J. B. Altepeter, and A. G. White, Science 290, 498 (2000).

[19] D. Kielpinski, V. Meyer, M. A. Rowe, C. A. Sackett, W. M. Itano, C. Monroe, and D. J. Wineland, Science 291, 1013 (2001).

[20] V. Paulisch, H. J. Kimble, and A. González-Tudela, New J. Phys. 18, 043041 (2016).

[21] M. L. Juan, R. Gordon, Y. Pang, F. Eftekhari, and R. Quidant, Nat. Phys. 5, 915 (2009).

[22] A. G. Brolo, Nat. Photonics 6, 709 (2012).

[23] E. Betzig and J. K. Trautman, Science 257, 189 (1992).

[24] N. Tischler, I. Fernandez-Corbaton, X. Zambrana-Puyalto, A. Minovich, X. Vidal, M. L. Juan, and G. Molina-Terriza, Light Sci. Appl. 3, e183 (2014).

[25] I. Fernandez-Corbaton, X. Zambrana-Puyalto, and G. Molina-Terriza, Phys. Rev. A 86, 042103 (2012).

[26] I. Fernandez-Corbaton, X. Zambrana-Puyalto, N. Tischler, X. Vidal, M. L. Juan, and G. Molina-Terriza,Phys. Rev. Lett. 111, 060401 (2013).

[27] K. Y. Bliokh, E. A. Ostrovskaya, M. A. Alonso, O. G. Rodríguez-Herrera, D. Lara, and C. Dainty, Opt. Express 19, 26132 (2011).

[28] Y. Gorodetski, N. Shitrit, I. Bretner, V. Kleiner, and E. Hasman, Nano Lett. 9, 3016 (2009).
[29] J. Petersen, J. Volz, and A. Rauschenbeutel, Science 346, 67 (2014).

[30] N. Tischler, C. Rockstuhl, and K. Słowik,Phys. Rev. X 8, 021017 (2018).

[31] B. Vest, M.-C. Dheur, E. Devaux, A. Baron, E. Rousseau, J.-P. Hugonin, J.-J. Greffet, G. Messin, and F. Marquier, Science 356, 1373 (2017).

[32] K. Mattle, H. Weinfurter, P. G. Kwiat, and A. Zeilinger, Phys. Rev. Lett. 76, 4656 (1996).

[33] S. P. Walborn, W. A. T. Nogueira, S. Pádua, and C. H. Monken, Europhys. Lett. 62, 161 (2003).

[34] See Supplemental Material at http://link.aps.org/ supplemental/10.1103/PhysRevLett.121.173901 for details on the source and experimental configuration.

[35] R. B. A. Adamson, L. K. Shalm, M. W. Mitchell, and A. M. Steinberg, Phys. Rev. Lett. 98, 043601 (2007).

[36] F. Wolfgramm, C. Vitelli, F. A. Beduini, N. Godbout, and M. W. Mitchell, Nat. Photonics 7, 28 (2013).

[37] L. Marrucci, C. Manzo, and D. Paparo, Phys. Rev. Lett. 96, 163905 (2006).

[38] E. Nagali, F. Sciarrino, F. De Martini, L. Marrucci, B. Piccirillo, E. Karimi, and E. Santamato, Phys. Rev. Lett. 103, 013601 (2009).

[39] M. B. Plenio and S. Virmani, Quantum Inf. Comput. 7, 1 (2007).

[40] F. Verstraete, K. Audenaert, J. Dehaene, and B. De Moor, J. Phys. A 34, 10327 (2001).

[41] D. Bruß, Phys. Rev. A 60, 4344 (1999). 